\title{
CDO term structure modelling with Lévy processes and the relation to market models*
}

\author{
Thorsten Schmidt ${ }^{\dagger}$ and Jerzy Zabczyk $\ddagger$
}

November 18, 2018

\begin{abstract}
This paper considers the modelling of collateralized debt obligations (CDOs). We propose a top-down model via forward rates generalizing Filipović, Overbeck and Schmidt (2009) to the case where the forward rates are driven by a finite dimensional Lévy process. The contribution of this work is twofold: we provide conditions for absence of arbitrage in this generalized framework. Furthermore, we study the relation to market models by embedding them in the forward rate framework in spirit of Brace, Gatarek, and Musiela (1997)

Key words: collateralized debt obligations, loss process, single tranche CDO, term structure of forward spreads, Levy processes, market models, Libor rate.
\end{abstract}

\section{Introduction}

A collateralized debt obligation (CDO) is a security backed by a pool of credit risky securities. There are issued notes on so-called tranches of the CDO which are characterized by different levels of credit riskiness or, in financial terms, seniorities. In this work we consider a general model for the evolution of tranche prices similar in spirit to the forward rate approach of Heath, Jarrow, and Morton (1992) and derive conditions under which the model is free of arbitrage.

For practical applications, market models are of a high importance. In this kind of models, traded securities have a finite set of maturities while in the forward rate approach all possible maturities are considered. For the pricing of options respectivly calibration of the model one imposes a simple dynamics for suitable quantities and uses the conditions for absence of arbitrage which results in tractable pricing formulas.

The main goal of this work is to provide conditions which guarantee the absence of arbitrage in a general class of models and to study market models embedded in this framework. The new contribution of this work is the following: first, we consider models where the driving random process is a Lévy process, generalizing Filipović, Overbeck, and Schmidt (2010). We derive general drift conditions which ensure that the market is free of arbitrage. In a next step we consider market models similar to those in Eberlein, Grbac, and Schmidt (2010). However, we embed the

\footnotetext{
* Support by the Polish Ministry of Science and Education projects: 5 1P03A 03429 "Stochastic evoluation equations with Lévy noise" and N N201 419039 "Stochastic equations in infinite dimensional spaces" is gratefully acknowledged. We thank the Fields Institute for the kind support as well as Frank Gehmlich and an anonymous referee for their helpful comments.

${ }^{\dagger}$ Technical University Chemnitz, Reichenhainer Str. 41, 09126 Chemnitz, Germany. Email: thorsten.schmidt@mathematik.tu-chemnitz.de

${ }^{\ddagger}$ Institute of Mathematics of the Polish Academy of Sciences, Sniadeckich 8, P.O.B. 21, 00-956 Warszawa 10, Poland
} 
market models in the forward rate models and derive the resulting drift conditions. The risk-free case studied in Brace, Gatarek, and Musiela (1997) turns out to be a special case.

\section{Collateralized Debt Obligations}

Consider a complete filtered probability space $\left(\Omega, \mathcal{F},\left(\mathcal{F}_{t}\right)_{t \geq 0}, \mathbb{P}\right)$ satisfying the usual conditions. Mathematically speaking, a collateralized debt obligation is a derivative on a portfolio of $N$ credit risky securities. With each security there is an associated nominal and we assume that the total nominal is one. Denote the process of accumulated losses over time by $L=\left(L_{t}\right)_{t \geq 0}$. Then $L$ is a pure-jump process which jumps upward at defaults of the securities in the pool and the jump size is the occurring loss. As the total nominal is one, $L_{t} \in[0,1]$ for all $t \geq 0$. A special case, quite often considered in practice, is when the loss on each default is a constant. By $\mathcal{I} \subset[0,1]$ we denote the set of attainable loss fractions and we assume that $\mathcal{I}=[0,1]$. The case where $\mathcal{I}$ is finite may be considered analogously, see Filipović, Overbeck, and Schmidt (2010).

Similar to Filipović, Overbeck, and Schmidt (2010) we consider $(T, x)$-bonds as basic constituents: a $(T, x)$-bond pays $1_{\left\{L_{T} \leq x\right\}}$ at maturity $T$, for $x \in \mathcal{I}$. Its price at time $t$ is denoted by $P(t, T, x)$. For $x=1$ we obtain that $P(t, T, 1)=: P(t, T)$ equals the risk-free bond. In Eberlein, Grbac, and Schmidt (2010), Section 6.1, it is shown how to extract $(T, x)$-bonds from observed CDO quotes under common assumptions.

Pricing CDOs with $(\mathbf{T}, \mathbf{x})$-bonds. We consider $(T, x)$-bonds as they are sufficient to provide prices for CDOs and similar derivatives as we will show in this section. First of all, $(T, x)$-bonds are sufficient for pricing European derivatives on the loss process. Indeed, consider a payoff $h\left(L_{T}\right)$ such that

$$
h\left(L_{T}\right)=h(1)-\int_{0}^{1} h^{\prime}(y) 1_{\left\{L_{T} \leq y\right\}} d y .
$$

Linearity of prices on contingent claims implies that the price at time $t$ of the derivative offering $h\left(L_{T}\right)$ at $T$ is given by the following functional of $(T, x)$-bonds

$$
h(1) P(t, T)-\int_{0}^{1} h^{\prime}(y) P(t, T, y) d y .
$$

A more general result holds true. Investing in CDOs is done via a so-called singletranche CDO (STCDO), sometimes also called tranche credit default swap. A STCDO is represented by its lower and upper detachment points, $x_{1}$ and $x_{2}$, with $0 \leq x_{1}<x_{2} \leq 1$. The investor receives coupon payments at times $T_{1}, \ldots, T_{n}$. In exchange to this, the investor covers a certain part of the occurring losses in each period. Set

$$
H(x):=\left(x_{2}-x\right)^{+}-\left(x_{1}-x\right)^{+}=\int_{\left(x_{1}, x_{2}\right]} 1_{\{x \leq y\}} d y .
$$

Then, investing in the STCDO with swap rate $S$ is equivalent to the following payment stream:

1. Payment leg. The investor receives $S H\left(L_{T_{i}}\right)$ at times $T_{i}, i=1, \ldots, n$. 
2. Default leg. The investor pays $-d H\left(L_{t}\right)=H\left(L_{t-}\right)-H\left(L_{t}\right)$ at any time where $\Delta L_{t} \neq 0$.

In Filipović, Overbeck, and Schmidt (2010) it is shown that the value of the STCDO at time $t$ can be derived solely on the basis of $(T, x)$-bonds. In the case where riskfree and risky $(T, x)$-bonds are independent, it follows from Lemma 4.1 therein that the value of the STCDO at time $t$ is given by

$$
\begin{gathered}
V(t, S)=\int_{\left(x_{1}, x_{2}\right]}\left(S \sum_{i=1}^{n} P\left(t, T_{i}, y\right)+P\left(t, T_{n}, y\right)-P\left(t, T_{0}, y\right)\right. \\
\left.+\int_{T_{0}}^{T_{n}} f(t, u) P(t, u, y) d u\right) d y,
\end{gathered}
$$

where $f(t, u)$ denotes the risk-free forward rate. Setting $V=0$ and solving for $S$ gives the par-spread for this investment. Market prices of STCDOs are typically quoted via the par-spread.

\section{Arbitrage-free term structure movements}

In this article we consider term structure movements of $(T, x)$-bonds given by

$$
P(t, T, x)=1_{\left\{L_{t} \leq x\right\}} \exp \left(-\int_{t}^{T} f(t, u, x) d u\right),
$$

where $f(t, T, x)$ is the $(T, x)$-forward rate prevailing at $t$. Let us assume that

(A1) $L_{t}=\sum_{s \leq t} \Delta L_{s}$ is càdlàg, non-decreasing, adapted, pure jump process, which admits an absolutely continuous compensator $\nu^{L}(t, d x) d t$ satisfying $\int_{0}^{t} \nu^{L}(s, \mathcal{I}) d s<$ $\infty$ (finite activity).

As shown in Filipović, Overbeck, and Schmidt (2010), under (A1), the indicator process $\left(1_{\left\{L_{t} \leq x\right\}}\right)_{t \geq 0}$ is cádlág and has intensity

$$
\lambda(t, x)=\nu^{L}\left(t,\left(x-L_{t}, 1\right]\right) .
$$

That is,

$$
M_{t}^{x}=1_{\left\{L_{t} \leq x\right\}}+\int_{0}^{t} 1_{\left\{L_{s} \leq x\right\}} \lambda(s, x) d s
$$

is a martingale. Moreover, $\lambda(t, x)$ is decreasing and continuous in $x$ with $\lambda(t, 1)=0$.

Consider a $d$-dimensional Lévy process $Z$. Denote by $\langle\cdot, \cdot\rangle$ the scalar product in $\mathbb{R}^{d}$, by $\cdot^{\top}$ the transpose and by $|\cdot|$ the respective norm on $\mathbb{R}^{d}$. It is well-known that a Lévy-process can be decomposed as

$$
Z_{t}=m t+W_{t}+\int_{0}^{t} \int_{|z| \leq 1} z(\mu(d s, d z)-\nu(d z) d s)+\int_{0}^{t} \int_{|z|>1} z \mu(d s, d z)
$$

where $m \in \mathbb{R}^{d}, W$ is a $d$-dimensional Wiener process with covariance matrix $\Sigma$ and $\mu$ is the random measure of jumps with its $\mathbb{P}$-compensator $\nu(d z) d s$. That is, for any 
Borel set $B$ of $\mathbb{R}^{+}$and any Borel set $\Lambda$ of $\mathbb{R}^{d}, \mu$ denotes the number of jumps in the time interval $B$ which have sizes in $\Lambda$,

$$
\mu(\omega ; B, \Lambda)=\sum_{s \in B} 1_{\Lambda}\left(\Delta Z_{s}\right) .
$$

The process $Z$ has exponential moments if the Laplace transform is always finite. Then the Laplace transform satisfies $\mathbb{E}\left(e^{-\left\langle u, Z_{t}\right\rangle}\right)=e^{t J(u)}$ for all $u \in \mathbb{R}^{d}$ with

$$
J(u)=-\langle m, u\rangle+\frac{1}{2}\langle\Sigma u, u\rangle+\int_{\mathbb{R}^{d}}\left(e^{-\langle u, z\rangle}-1+1_{\{|z| \leq 1\}}(z)\langle u, z\rangle\right) \nu(d z) .
$$

We assume throughout that the $(T, x)$-forward rate is given by

$$
\begin{aligned}
f(t, T, x)=f(0, T, x) & +\int_{0}^{t} a(s, T, x) d s+\int_{0}^{t}\left\langle b(s, T, x), d Z_{s}\right\rangle \\
& +\int_{0}^{t} \int_{\mathcal{I}} c(s, T, x ; y) \mu^{L}(d s, d y)
\end{aligned}
$$

where $\mu^{L}$ is the random measure associated to the jumps of $L$. By $[L, Z]$ we denote the covariation of $L$ and $Z$. Let

$$
B:=\left\{u \in \mathbb{R}^{d}: \int_{|z|>1} e^{-\langle u, z\rangle} \nu(d z)<\infty\right\} .
$$

Additionally, we use the following assumptions.

(A2) For each $T, x$ the processes $a, b$, and $c$ are assumed to be predictable and have bounded trajectories. Furthermore, $c(\cdot, T, 1 ; y)=0$ for all $y \in \mathcal{I}$.

(A3) $[L, Z]_{t}=0$ for all $t \geq 0$.

(A4) For any $r>0$ the function $u \mapsto \int_{|z|>1} e^{-\langle u, z\rangle} \nu(d z)$ is bounded on $\left\{u \in \mathbb{R}^{d}\right.$ : $|u| \leq r\} \cap B$.

(A5) For each $(T, x)$ there exists $K(T, x)$ such that

$$
\sup _{0 \leq s \leq T, y \in \mathbb{R}^{d}} c(s, T, x ; y) \leq K(T, x) .
$$

The assumption on $c$ in (A2) states that losses in the considered portfolio do not influence the risk-free rate. This assumption can be relaxed but at the cost of further notation. Assumption (A3) is natural from a modelling point of view: jumps in $L$ influence $f$ only through $c$ and not via a dependence of $L$ and $Z$.

Contagion. This framework allows for two kinds of contagion, i.e. feedback of the loss process $L$ on the forward rates: first, a direct contagion via simultaneous jumps of $L$ and $f$; when $\Delta L_{t} \neq 0$, (A3) gives that $\Delta f(t, T, x)=c\left(t, T, x ; \Delta L_{t}\right)$. Second, a kind of indirect contagion via letting the model parameters $a, b$, and $c$ be explicit functions of the loss path $L$.

It is well-known that then the market of $(T, x)$-bonds is free of arbitrage if

$$
\left(D_{t} P(t, T, x)\right)_{0 \leq t \leq T} \text { are local martingales for all }(T, x),
$$

where $D$ is the discounting process given by

$$
D_{t}=e^{-\int_{0}^{t} r_{s} d s}=e^{-\int_{0}^{t} f(s, s, 1) d s} .
$$

The goal of this section is to give conditions which are sufficient for (7) to hold. 
Default-free market. The default-free forward rate, $f(t, T)$, is given by

$$
f(t, T)=f(t, T, 1) .
$$

We also denote $a(t, T)=a(t, T, 1)$ and $b(t, T)=b(t, T, 1)$. In the case of default free markets, the following was shown in Jakubowski and Zabczyk (2007), Theorem 3.1. On one side, (A2) and the no-arbitrage condition

$$
D_{t} P(t, T) \text { are local martingales for all } 0 \leq t \leq T \text {; }
$$

imply that for any $u \leq T$

$$
\int_{t}^{u} b(t, v) d v \in B \quad \text { for almost all } t \in[0, u] .
$$

On the other side, if (A2), (A3) and (9) hold, then (8) is equivalent to

$$
\int_{t}^{s} a(t, u) d u=J\left(\int_{t}^{s} b(t, u) d u\right)
$$

for almost all $0 \leq t \leq s \leq T$.

The drift conditions. Now we are in the position to state the model for the defaultable market and derive the drift conditions. Recall that we consider a market consisting of $(T, x)$-bonds.

Theorem 3.1. Assume that (A1)-(A4) hold.

(i) If (A5) holds, it follows from (7) that

$$
\int_{t}^{s} b(t, v, x) d v \in B
$$

for any $0 \leq t \leq s$ on $\left\{L_{t} \leq x\right\}, \mathbb{Q} \otimes d t$-a.s.

(ii) If (11) holds, then (77) is equivalent to

$$
\begin{aligned}
\int_{t}^{s} a(t, u, x) d u & =J\left(\int_{t}^{s} b(t, u, x) d u\right) \\
& +\int_{\mathcal{I}}\left(e^{-\int_{t}^{s} c(t, u, x ; y) d u}-1\right) 1_{\left\{L_{t}+y \leq x\right\}} \nu^{L}(t, d y) \\
f(t, t, x) & =f(t, t)+\lambda(t, x)
\end{aligned}
$$

for any $0 \leq t \leq s$, where (12) and (13) hold on $\left\{L_{t} \leq x\right\}, \mathbb{Q} \otimes d t$-a.s.

Proof. Set

$$
p(t, T, x):=\exp \left(-\int_{t}^{T} f(t, u, x) d u\right),
$$


such that $P(t, T, x)=1_{\left\{L_{t} \leq x\right\}} p(t, T, x)$. Recall the martingale $M^{x}$ from (3). Then

$$
\begin{aligned}
d\left(D_{t} P(t, T, x)\right) & =D_{t} p(t-, T, x) d M_{t}^{x}-D_{t} p(t-, T, x) \lambda(t, x) 1_{\left\{L_{t} \leq x\right\}} d t \\
& +1_{\left\{L_{t-} \leq x\right\}} d\left(D_{t} p(t, T, x)\right)+d\left[1_{\left\{L_{t} \leq x\right\}}, D_{t} p(t, T, x)\right] .
\end{aligned}
$$

Denote $a^{*}(t, T, x):=\int_{t}^{T} a(t, u, x) d u, b^{*}(t, T, x):=\int_{t}^{T} b(t, u, x) d u$, and similarly $c^{*}(t, T, x ; y):=\int_{t}^{T} c(t, u, x ; y) d u$. We first compute the last term in (14). Note that the process $\left(1_{\left\{L_{t} \leq x\right\}}\right)_{t \geq 0}$ jumps at most once, from 1 to 0 , at the first time when $L$ crosses the barrier $x$. Hence,

$$
\begin{aligned}
{\left[1_{\{L, \leq x\}},\right.} & D \cdot p(\cdot, T, x)]_{t}=\sum_{0 \leq s \leq t} \Delta 1_{\left\{L_{s} \leq x\right\}} \Delta\left(D_{s} p(s, T, x)\right) \\
= & -\sum_{0 \leq s \leq t} 1_{\left\{L_{s-} \leq x, L_{s}>x\right\}} \Delta\left(D_{s} p(s, T, x)\right) \\
= & -\int_{0}^{t} \int_{\mathcal{I}} 1_{\left\{L_{s-} \leq x\right\}} D_{s} p(s-, T, x) 1_{\left\{L_{s-}+y>x\right\}}\left(e^{-c^{*}(s, T, x ; y)}-1\right) \mu^{L}(d s, d y),
\end{aligned}
$$

where we used in the last step that by (A3), $Z$ and $L$ do not have simultaneous jumps. Regarding the remaining term in (14), we obtain from (11) and (6), by the Itô -formula, that

$$
\begin{aligned}
1_{\left\{L_{t-} \leq x\right\}} d\left(D_{t} p(t, T, x)\right) & =D_{t} p(t-, T, x) 1_{\left\{L_{t-} \leq x\right\}} \\
& \left(\left(f(t, t, x)-r_{t}-\left\langle b^{*}(t, T, x), m\right\rangle-a^{*}(t, T, x)\right) d t\right. \\
& +\frac{1}{2} b^{*}(t, T, x)^{\top} \Sigma b^{*}(t, T, x) d t \\
& +\int_{\mathbb{R}^{d}}\left[e^{-b^{*}(t, T, x)^{\top} z}-1+b^{*}(t, T, x)^{\top} z\right] 1_{\{|z| \leq 1\}} \mu(d t, d z) \\
& +\int_{\mathbb{R}^{d}}\left[e^{-b^{*}(t, T, x)^{\top} z}-1\right] 1_{\{|z|>1\}} \mu(d t, d z) \\
& \left.+\int_{\mathcal{I}} 1_{\left\{L_{t-} \leq x\right\}}\left[e^{-c^{*}(t, T, x ; y)}-1\right] \mu^{L}(d t, d y)\right) \\
& +d \tilde{M}_{t}
\end{aligned}
$$

where $\tilde{M}$ is a local martingale and we used the decomposition (44). Inserting (15) 
and (16) in (14) we obtain, using $1_{\left\{L_{t-\leq x}\right\}} p(t-, T, x)=P(t-, T, x)$, that

$$
\begin{aligned}
d\left(D_{t} P(t, T, x)\right) & =D_{t} P(t-, T, x) \\
& \left(d M_{t}^{x}-\lambda(t, x) 1_{\left\{L_{t} \leq x\right\}} d t\right. \\
& \left(f(t, t, x)-r_{t}-\left\langle b^{*}(t, T, x), m\right\rangle-a^{*}(t, T, x)\right) d t \\
& +\frac{1}{2} b^{*}(t, T, x)^{\top} \Sigma b^{*}(t, T, x) d t \\
& +\int_{\mathbb{R}^{d}}\left[e^{-b^{*}(t, T, x)^{\top} z}-1+b^{*}(t, T, x)^{\top} z\right] 1_{\{|z| \leq 1\}} \mu(d t, d z) \\
& +\int_{\mathbb{R}^{d}}\left[e^{-b^{*}(t, T, x)^{\top} z}-1\right] 1_{\{|z|>1\}} \mu(d t, d z) \\
& \left.+\int_{\mathcal{I}} 1_{\left\{L_{t-}+y \leq x\right\}}\left(e^{-c^{*}(t, T, x ; y)}-1\right) \mu^{L}(d t, d y)\right)+d \tilde{\tilde{M}}_{t}
\end{aligned}
$$

with a local martingale $\tilde{\tilde{M}}$. First we study (i). From (17) we obtain that

$$
\begin{aligned}
D_{t} P(t, T, x) & =D_{0} P(0, T, x)+A(t)+\tilde{\tilde{\tilde{M}}}_{t} \\
& +\int_{0}^{t} \int_{\mathbb{R}^{d}} D_{s} P(s-, T, x)\left(e^{-b^{*}(s, T, x)^{\top} z}-1+b^{*}(s, T, x)^{\top} z\right) 1_{\{|z| \leq 1\}} \mu(d s, d z) \\
& +\int_{0}^{t} \int_{\mathbb{R}^{d}} D_{s} P(s-, T, x)\left(e^{-b^{*}(s, T, x)^{\top} z}\right) 1_{\{|z|>1\}} \mu(d s, d z) \\
& -\int_{0}^{t} \int_{\mathbb{R}^{d}} D_{s} P(s-, T, x) 1_{\{|z|>1\}} \mu(d s, d z) \\
& \left.+\int_{0}^{t} \int_{\mathcal{I}} D_{s} P(s-, T, x) 1_{\left\{L_{s-}+y \leq x\right\}}\left(e^{-c^{*}(s, T, x ; y)}-1\right) \mu^{L}(d s, d y)\right) \\
= & : D_{0} P(0, T, x)+A(t)+\tilde{\tilde{M}}_{t}+I_{1}(t)+I_{2}(t)-I_{3}(t)+I_{4}(t) .
\end{aligned}
$$

Here $\tilde{\tilde{M}}_{t}$ is a local martingale and $A(t)$ is a $d t$ integral and thus they both are locally integrable processes. The no-arbitrage condition (7) implies that the sum of all those six processes is a local martingale and in particular the sum

$$
I_{1}(t)+I_{2}(t)-I_{3}(t)+I_{4}(t)
$$

is locally integrable. We consider each term separately. Since $\left(D_{t} P(t, T, x)\right)_{0 \leq t \leq T}$ is a nonnegative local martingale, it is a supermartingale and hence

$$
\sup _{0 \leq s \leq T} \mathbb{E}\left(D_{s} P(s-, T, x)\right) \leq \sup _{0 \leq s \leq T} \mathbb{E}\left(D_{s} P(s, T, x)\right) \leq \mathbb{E}\left(D_{0} P(0, T, x)\right)<\infty
$$

the last expectation being finite by (7). The process

$$
I_{3}(t):=\int_{0}^{t} \int_{\mathbb{R}^{d}} D_{s} P(s-, T, x) 1_{\{|z|>1\}} \mu(d s, d z)
$$


is locally integrable. To see this let $\left(\tau_{n}\right)$ be an increasing sequence of stopping times dominated by $T$. Then

$$
\begin{aligned}
\mathbb{E}\left(\int_{0}^{\tau_{n}} \int_{\mathbb{R}^{d}} D_{s} P(s-, T, x) 1_{\{|z|>1\}} \mu(d s, d z)\right) \\
\leq \mathbb{E}\left(\int_{0}^{T} \int_{\mathbb{R}^{d}} D_{s} P(s-, T, x) 1_{\{|z|>1\}} \mu(d s, d z)\right) \\
\quad=\int_{0}^{T} \int_{\mathbb{R}^{d}} \mathbb{E}\left(D_{s} P(s-, T, x)\right) 1_{\{|z|>1\}} \nu(d z) d s .
\end{aligned}
$$

Since the integrands are nonnegative, we obtain with (19) that

$\mathbb{E}\left(\int_{0}^{\tau_{n}} \int_{\mathbb{R}^{d}} D_{s} P(s-, T, x) 1_{\{|z|>1\}} \mu(d s, d z)\right) \leq T \mathbb{E}\left(D_{0} P(0, T, x)\right) \nu\left(\left\{z \in \mathbb{R}^{d}:|z|>1\right\}\right)<\infty$.

Next, we consider $I_{4}$. By (A5)

$$
\left|e^{-c^{*}(s, T, x ; y)}-1\right| \leq e^{T K(T, x)}+1=: \tilde{K}(T, x)
$$

and hence

$$
\begin{aligned}
& \mathbb{E}\left(\int_{0}^{\tau_{n}} \int_{\mathbb{R}^{d}} D(s) P(s-, T, x) 1_{\left\{L_{s-}+y \leq x\right\}}\left|e^{-c^{*}(s, T, x ; y)}-1\right| \mu^{L}(d s, d y)\right) \\
& \quad \leq \tilde{K}(T, x) \mathbb{E}\left(\int_{0}^{T} \int_{\mathbb{R}^{d}} 1_{\left[0, \tau_{n}\right]}(s) D(s) P(s-, T, x) 1_{\left\{L_{s-}+y \leq x\right\}} \mu^{L}(d s, d y)\right) \\
& \quad \leq \tilde{K}(T, x) \int_{0}^{T} \int_{\mathbb{R}^{d}} \mathbb{E}(D(s) P(s-, T, x)) \nu^{L}(s, d y),
\end{aligned}
$$

which is finite by (19), (A1) and the second part in (A5). Summarizing, the noarbitrage condition (7) implies that the sum $I_{1}+I_{2}$ is locally integrable. However

$$
\begin{aligned}
I_{1}+I_{2} & =\int_{0}^{t} \int_{\mathbb{R}^{d}}\left(e^{-b^{*}(s, T, x)^{\top} z}-1+b^{*}(s, T, x)^{\top} z\right) 1_{\{|z| \leq 1\}} \mu(d s, d z) \\
& +\int_{0}^{t} \int_{\mathbb{R}^{d}} e^{-b^{*}(s, T, x)^{\top} z} 1_{\{|z|>1\}} \mu(d s, d z) .
\end{aligned}
$$

Since both integrands are nonnegative, this sum is locally integrable if and only if both summands are locally integrable and so $\int_{0}^{t} \int_{\mathbb{R}^{d}} e^{-b^{*}(s, T, x)^{\top} z} 1_{\{|z|>1\}} \mu(d s, d z)$ is locally integrable. Therefore, for a localizing sequence $\tau_{n}$

$$
\mathbb{E} \int_{0}^{T} \int_{\mathbb{R}^{d}} e^{-b^{*}(s, T, x)^{\top} z} 1_{\left\{s \leq \tau_{n}\right\}} 1_{\{|z|>1\}} \mu(d s, d z)<+\infty .
$$


Equivalently

$$
\mathbb{E}\left[\int_{0}^{\tau_{n}}\left(\int_{|z|>1} e^{-b^{*}(s, T, x)^{\top} z} \nu(d z)\right) d s\right]<+\infty .
$$

This implies (11) and assertion (i) follows.

We consider now (ii). Note that $D_{t} p(t-, T, x)>0$. Compensating in (16) the integrals with respect to the random measures $\mu$ and $\mu^{L}$, collecting the 'dt'-terms and dividing by $D_{t} p(t-, T, x)>0$ gives

$$
\begin{aligned}
& 1_{\left\{L_{t} \leq x\right\}}\left[f(t, t, x)-r_{t}-\lambda(t, x)-\left\langle b^{*}(t, T, x), m\right\rangle-a^{*}(t, T, x)\right. \\
&+ \frac{1}{2} b^{*}(t, T, x)^{\top} \Sigma b^{*}(t, T, x) \\
&+\int_{\mathbb{R}^{d}}\left[e^{-b^{*}(t, T, x)^{\top} z}-1+1_{\{|z| \leq 1\}} b^{*}(t, T, x)^{\top} z\right] \nu(d z) \\
&\left.+\int_{\mathcal{I}} 1_{\left\{L_{t}+y \leq x\right\}}\left(e^{-c^{*}(t, T, x ; y)}-1\right) \nu^{L}(t, d y)\right] d t .
\end{aligned}
$$

$D p$ being a local martingale is equivalent to having a vanishing drift. From (21) we obtain the following condition:

$$
\begin{aligned}
0 & =f(t, t, x)-r_{t}-\lambda(t, x)-a^{*}(t, T, x)+J\left(b^{*}(t, T, x)\right) \\
& +\int_{\mathcal{I}}\left(e^{-c^{*}(t, T, x ; y)}-1\right) 1_{\left\{L_{t}+y \leq x\right\}} \nu^{L}(t, d y)
\end{aligned}
$$

$d t \otimes \mathbb{Q}$-almost surely for all $T \geq t$. Let $N \in \mathcal{B}\left(\mathbb{R}^{+}\right) \otimes \mathcal{F}$ be the set of all $(t, \omega)$ for which (22) holds. For $(t, \omega) \in N$ we choose $T=t$ and hence obtain (13) as $J(0)=0$. The remaining terms give (12), where both equations hold only on $N$, i.e. $d t \otimes \mathbb{Q}$-almost surely.

For the converse, we need to show that the drift conditions imply that all discounted $(T, x)$-bonds are local martingales. For fixed $x$, the drift conditions imply that the 'dt'-terms in (17) vanish (compare (21)) and hence $\left(D_{t} P(t, T, x)\right)_{0 \leq t \leq T}$ are local martingales. The conclusion follows.

Theorem 3.1 states that, under the no-arbitrage condition (77), the drift $a(t, \cdot, x)$ of the forward rates is determined by the volatility $b(t, \cdot, x)$ and the contagion $c(t, \cdot, x ; \cdot)$. Besides this, (12) gives an implicit relation between $f(t, t, x)$ and $L_{t}$ or more precisely its compensator; the relationship between $\lambda$ and the compensator $\nu$ is given in (2).

In Filipović, Overbeck, and Schmidt (2010) it is shown how to construct a conditional Markov process which satisfies the drift conditions when the filtration is generated by a Brownian motion. The extension to the case where the background filtration is generated by a Lévy process is studied in Schmidt and Tappe (2010).

There is a rich literature on drift conditions in the defaultable and default-free case, see for example Björk, Di Masi, Kabanov, and Runggaldier (1997), Musiela and Rutkowski (1997), Filipović (2001), Schmidt (2006), Ozkan and Schmidt (2005) and Huehne (2007).

\section{Market Models}

For practical applications it is important to realize, that $(T, x)$-bond prices are neither available for all maturities nor for all levels $x$. This chapter is devoted to 
the study of a market model where this assumption is relaxed. We start by deriving dynamics of $\left(T_{k}, x_{i}\right)$-rates in section 4.1, and consider market models of the forward rate and the $\left(T_{k}, x_{i}\right)$-rate in sections 4.2 and 4.3 , respectively. The drift condition derived in Theorem 3.1 is, however, still sufficient for absence of arbitrage. Recall that $\left(L_{t}\right)_{t \geq 0}$ was the loss process of the CDO and $P(t, T, x)=1_{\left\{L_{t} \leq x\right\}} p(t, T, x)$ with $p(t, T, x)>0$.

We fix a tenor structure $0<T_{1}<\cdots<T_{n}$ and a barrier structure $0=x_{1}<$ $\cdots<x_{m}=1$. Set $\mathcal{S}:=\left\{T_{1}, \ldots, T_{n}\right\} \times\left\{x_{0}, \ldots, x_{m}\right\}$. The considered market model consists of all $(T, x)$-bonds with $(T, x) \in \mathcal{S}$. As $x_{m}=1$, the market also contains risk-free bonds. Throughout we assume that the initial term structure, denoted in $(T, x)$-bonds, is strictly positive and decreasing in $T$ and increasing in $x$.

Definition 4.1. Set $\delta_{k}:=T_{k+1}-T_{k}$. The rate

$$
L\left(t, T_{k}, x_{i}\right):=1_{\left\{L_{t} \leq x_{i}\right\}} \frac{1}{\delta_{k}}\left(\frac{p\left(t, T_{k}, x_{i}\right)}{p\left(t, T_{k+1}, x_{i}\right)}-1\right)
$$

is called $\left(T_{k}, x_{i}\right)$-rate. For all $0 \leq t \leq S \leq T$ and $x \in \mathcal{I}$,

$$
F(t, S, T, x):=1_{\left\{L_{t} \leq x\right\}} \frac{p(t, S, x)}{p(t, T, x)}
$$

defines the $(S, T, x)$-forward bond price.

The $\left(T_{k}, 1\right)$-rate is the so-called LIBOR-rate. It is a default-free and has been studied in many papers, see e.g. Filipović (2009). Defaultable LIBOR-rates have been studied in Eberlein, Kluge, and Schönbucher (2006), In this paper we follow Brace, Gatarek, and Musiela (1997) and embed the $\left(T_{k}, x_{i}\right)$-rates in the framework of continuous forward rates and obtain sufficient conditions for absence of arbitrage. In Eberlein, Grbac, and Schmidt (2010) $\left(T_{k}, x\right)$-rates are studied directly; however they do not consider a discrete structure for the loss levels.

\subsection{Dynamics of $\left(T_{k}, x_{i}\right)$-rates}

In this section we derive the dynamics of $\left(T_{k}, x_{i}\right)$-rates when the drift condition (12) is satisfied. This result generalizes Eberlein and Özkan (2005) and Huehne (2007). Recall that $b^{*}(t, T, x)=\int_{t}^{T} b(t, u, x) d u$.

Theorem 4.2. Assume that (A1)-(A4) and (12) hold and consider $\left(T_{k}, x_{i}\right) \in \mathcal{S}$. On $\left\{L_{t-} \leq x_{i}\right\}$ we have that

$$
\begin{aligned}
d L\left(t, T_{k}, x_{i}\right) & =\frac{1+\delta_{k} L\left(t-, T_{k}, x_{i}\right)}{\delta_{k}}\left(D\left(t, T_{k}, T_{k+1}, x_{i}\right)-\frac{\delta_{k} L\left(t-, T_{k}, x_{i}\right)}{1+\delta_{k} L\left(t-, T_{k}, x_{i}\right)} \lambda\left(t, x_{i}\right)\right) d t \\
& +d \tilde{M}_{t},
\end{aligned}
$$

where the drift term attributed to the compensated jumps and the quadratic variation of the diffusive part is

$$
\begin{aligned}
& D\left(t, T_{k}, T_{k+1}, x_{i}\right) \\
& :=\int_{\mathbb{R}^{d}}\left(e^{\left\langle b^{*}\left(t, T_{k+1}, x_{i}\right)-b^{*}\left(t, T_{k}, x_{i}\right), z\right\rangle}-e^{-\left\langle b^{*}\left(t, T_{k}, x_{i}\right), z\right\rangle}+e^{-\left\langle b^{*}\left(t, T_{k+1}, x_{i}\right), z\right\rangle}-1\right) \nu(d z) \\
& +\int_{\mathcal{I}}\left(e^{c^{*}\left(t, T_{k+1}, x_{i} ; y\right)-c^{*}\left(t, T_{k}, x_{i} ; y\right)}-e^{-c^{*}\left(t, T_{k}, x_{i} ; y\right)}+e^{-c^{*}\left(t, T_{k+1}, x_{i} ; y\right)}\right) 1_{\left\{L_{t}+y \leq x_{i}\right\}} \nu^{L}(t, d y) \\
& +\left\langle\Sigma\left(b^{*}\left(t, T_{k+1}, x_{i}\right)-b^{*}\left(t, T_{k}, x_{i}\right)\right), b^{*}\left(t, T_{k+1}, x_{i}\right)\right\rangle
\end{aligned}
$$


and $\tilde{M}=\tilde{M}\left(T_{k}, x_{i}\right)$ is the local martingale

$$
\begin{aligned}
d \tilde{M}_{t} & :=L\left(t-, T_{k}, x_{i}\right) d M_{t}^{x} \\
& +\frac{1+\delta_{k} L\left(t-, T_{k}, x_{i}\right)}{\delta_{k}}\left(\int_{\mathbb{R}^{d}}\left(e^{\left\langle b^{*}\left(t, T_{k+1}, x_{i}\right)-b^{*}\left(t, T_{k}, x_{i}\right), z\right\rangle}-1\right)(\mu(d s, d z)-\nu(d z) d s)\right. \\
& +\int_{\mathcal{I}}\left(e^{c^{*}\left(t, T_{k+1}, x_{i} ; y\right)-c^{*}\left(t, T_{k}, x_{i} ; y\right)}-1\right) 1_{\left\{L_{t-}+y \leq x_{i}\right\}}\left(\mu^{L}(d s, d y)-\nu^{L}(d y) d s\right) \\
& \left.+\left\langle b^{*}\left(t, T_{k+1}, x_{i}\right)-b^{*}\left(t, T_{k}, x_{i}\right), d W(t)\right\rangle\right) .
\end{aligned}
$$

Proof. Fix $0<S<T$. First, we derive the dynamics of the pre-default $(S, T, x)$ forward bond price process

$$
g(t, S, T, x):=\frac{p(t, S, x)}{p(t, T, x)} .
$$

With $x, S$ and $T$ fixed we denote $A(t):=\int_{S}^{T} a(t, u, x) d u, B(t):=\int_{S}^{T} b(t, u, x) d u$, and $C(t ; y):=\int_{S}^{T} c(t, u, x ; y) d u$. By (27) and the dynamics of the forward rate $f$ given in (6), the stochastic Fubini theorem yields

$$
\begin{aligned}
g(t, S, T, x) & =e^{\int_{S}^{T}\left(f(0, u, x)+\int_{0}^{t} a(s, u, x) d s+\int_{0}^{t}\left\langle b(s, u, x), d Z_{s}\right\rangle+\int_{0}^{t} \int_{\mathcal{I}} c(s, u, x ; y) \mu^{L}(d s, d y)\right) d u} \\
& =g(0, S, T, x) e^{\int_{0}^{t} A(s) d s+\int_{0}^{t}\langle B(s), d Z(s)\rangle+\int_{0}^{t} \int_{\mathcal{I}} C(s ; y) \mu^{L}(d s, d y)} .
\end{aligned}
$$

The Itô-formula gives an expression for the dynamics of $g$ :

$$
\begin{aligned}
d g(t, S, T, x) & =g(t-, S, T, x)(A(t)+\langle B(t), d Z(t)\rangle) d t \\
& +\frac{1}{2} g(t-, S, T, x)\langle\Sigma B(t), B(t)\rangle d t \\
& +(g(t, S, T, x)-g(t-, S, T, x)-g(t-, S, T, x)\langle B(t), \Delta Z(t)\rangle) \\
& =: d I_{1}(t)+d I_{2}(t)+d I_{3}(t) .
\end{aligned}
$$

The decomposition of $Z$ in (4) yields

$$
\begin{aligned}
d I_{1}(t) & =g(t-, S, T, x)(A(t)+\langle B(t), m\rangle) d t \\
& +g(t-, S, T, x)\langle B(t), d W(t)\rangle \\
& +\int_{\|z\| \leq 1} g(t-, S, T, x)\langle B(t), z\rangle(\mu(d t, d z)-\nu(d z) d t) \\
& +\int_{\|z\|>1} g(t-, S, T, x)\langle B(t), z\rangle \mu(d t, d z) .
\end{aligned}
$$

By (A3), $L$ and $Z$ have no joint jumps and therefore

$$
\begin{aligned}
d I_{3}(t) & =g(t-, S, T, x)\left(e^{\langle B(t), \Delta Z(t)\rangle+C(t, \Delta L(t))}-1\right)-g(t-, S, T, x)\langle B(t), \Delta Z(t)\rangle \\
& =\int_{\mathbb{R}^{d}} g(t-, S, T, x)\left(e^{\langle B(t), z\rangle}-1-\langle B(t), z\rangle\right) \mu(d t, d z) \\
& +\int_{\mathcal{I}} g(t-, S, T, x)\left(e^{C(t ; y)}-1\right) \mu^{L}(d t, d y)
\end{aligned}
$$


Inserting this expressions in (28) we obtain

$$
\begin{aligned}
\frac{d g(t, S, T, x)}{g(t-, S, T, x)} & =\left(A(t)+\langle B(t), m\rangle+\frac{1}{2}\langle\Sigma B(t), B(t)\rangle\right) d t+\langle B(t), d W(t)\rangle \\
& +\int_{\mathbb{R}^{d}}\left(e^{\langle B(t), z\rangle}-1\right)(\mu(d t, d z)-\nu(d z) d t) \\
& +\int_{\mathbb{R}^{d}}\left(e^{\langle B(t), z\rangle}-1-1_{\{\|z\| \leq 1\}}\langle B(t), z\rangle\right) \nu(d z) d t \\
& +\int_{\mathcal{I}}\left(e^{C(t ; y)}-1\right) \mu^{L}(d t, d y) .
\end{aligned}
$$

As $L\left(t, T_{k}, x\right)=1_{\left\{L_{t} \leq x\right\}} \delta_{k}^{-1}\left(g\left(t, T_{k}, T_{k+1}, x\right)-1\right)$, we have that

$$
\begin{aligned}
d L\left(t, T_{k}, x\right) & =\delta_{k}^{-1}\left(g\left(t-, T_{k}, T_{k+1}, x\right)-1\right) d 1_{\left\{L_{t} \leq x\right\}} \\
& +1_{\left\{L_{t-} \leq x\right\}} \delta_{k}^{-1} d g\left(t, T_{k}, T_{k+1}, x\right) \\
& +d\left[1_{\left\{L_{t} \leq x\right\}}, \delta_{k}^{-1}\left(g\left(t, T_{k}, T_{k+1}, x\right)-1\right)\right] .
\end{aligned}
$$

Note that $d 1_{\left\{L_{t} \leq x\right\}}=d M_{t}^{x}-1_{\left\{L_{t} \leq x\right\}} \lambda(t, x) d t$ and, similar to (15),

$$
\begin{aligned}
d\left[1_{\left\{L_{t} \leq x\right\}},\right. & \left.\delta_{k}^{-1}\left(g\left(t, T_{k}, T_{k+1}, x\right)-1\right)\right] \\
& =\delta_{k}^{-1} \int_{\mathcal{I}}\left(1_{\left\{L_{t-}+y \leq x\right\}}-1_{\left\{L_{t-} \leq x\right\}}\right) g\left(t-, T_{k}, T_{k+1}, x\right)\left(e^{C\left(t, T_{k}, T_{k+1} ; y\right)}-1\right) \mu^{L}(d t, d y) .
\end{aligned}
$$

Summarizing, we obtain the following dynamics of $L$,

$$
\begin{aligned}
d L\left(t, T_{k}, x\right) & =L\left(t-, T_{k}, x\right)\left(d M_{t}^{x}-\lambda(t, x) d t\right) \\
& +1_{\left\{L_{t-} \leq x\right\}} \frac{\delta_{k} L\left(t-, T_{k}, x\right)+1}{\delta_{k}}\left[\left(A(t)+\langle B(t), m\rangle+\frac{1}{2}\langle\Sigma B(t), B(t)\rangle\right.\right. \\
& \left.+\int_{\mathbb{R}^{d}}\left(e^{\langle B(t), z\rangle}-1-1_{\{\|z\| \leq 1\}}\langle B(t), z\rangle\right) \nu(d z)\right) d t \\
& +\langle B(t), d W(t)\rangle+\int_{\mathbb{R}^{d}}\left(e^{\langle B(t), z\rangle}-1\right)(\mu(d t, d z)-\nu(d z) d t) \\
& +\int_{\mathcal{I}}\left(e^{C(t ; y)}-1\right) \mu^{L}(d y, d t) \\
& \left.+\int_{\mathcal{I}}\left(1_{\left\{L_{t-}+y \leq x\right\}}-1_{\left\{L_{t-} \leq x\right\}}\right)\left(e^{C(t ; y)}-1\right) \mu^{L}(d t, d y)\right] .
\end{aligned}
$$

Compensating the remaining $\mu^{L}$ terms and collection all $d s$-terms and using (5) gives the drift term of $L$,

$$
\begin{aligned}
& -L\left(t-, T_{k}, x\right) \lambda(t, x) \\
& +1_{\left\{L_{t-} \leq x\right\}} \frac{\delta_{k} L\left(t-, T_{k}, x\right)+1}{\delta_{k}}\left[A(t)+J(-B(t))+\int_{\mathcal{I}}\left(e^{C(t ; y)}-1\right) 1_{\left\{L_{t}+y \leq x\right\}} \nu^{L}(t, d y)\right] .
\end{aligned}
$$

In the next step we will use the drift condition (12) to work further on this expression. Recall that

$$
B(t)=\int_{S}^{T} b(t, u, x) d u=b^{*}(t, T, x)-b^{*}(t, S, x),
$$


such that (5) gives

$$
\begin{aligned}
J(-B(t)) & =-\left\langle m, b^{*}(t, S, x)\right\rangle+\left\langle m, b^{*}(t, T, x)\right\rangle \\
& +\frac{1}{2}\left\langle\Sigma\left(b^{*}(t, T, x)-b^{*}(t, S, x)\right),\left(b^{*}(t, S, x)-b^{*}(t, T, x)\right)\right\rangle \\
& +\int_{\mathbb{R}^{d}}\left(e^{-\left\langle b^{*}(t, S, x)-b^{*}(t, T, x), z\right\rangle}-1+1_{\{\|z\| \leq 1\}}\left\langle b^{*}(t, S, x)-b^{*}(t, T, x), z\right\rangle\right) \nu(d z) \\
& =J\left(b^{*}(t, S, x)\right)-J\left(b^{*}(t, T, x)\right) \\
& +\left\langle\Sigma\left(b^{*}(t, T, x)-b^{*}(t, S, x)\right), b^{*}(t, T, x)\right\rangle \\
& +\int_{\mathbb{R}^{d}}\left(e^{-\left\langle b^{*}(t, S, x)-b^{*}(t, T, x), z\right\rangle}-e^{-\left\langle b^{*}(t, S, x), z\right\rangle}+e^{-\left\langle b^{*}(t, T, x), z\right\rangle}-1\right) \nu(d z)
\end{aligned}
$$

With $A(t)=a^{*}(t, T, x)-a^{*}(t, S, x)$ the drift condition (12) gives

$$
\begin{aligned}
A(t) & =J\left(b^{*}(t, T, x)\right)-J\left(b^{*}(t, S, x)\right) \\
& +\int_{\mathcal{I}}\left(e^{-c^{*}(t, T, x ; y)}-e^{-c^{*}(t, S, x ; y)}\right) 1_{\left\{L_{t}+y \leq x\right\}} \nu^{L}(t, d y) .
\end{aligned}
$$

Hence,

$$
\begin{aligned}
A(t) & +J(-B(t))+\int_{\mathcal{I}}\left(e^{C(s ; y)}-1\right) 1_{\left\{L_{t}+y \leq x\right\}} \nu^{L}(t, d y) \\
= & \left\langle\Sigma\left(b^{*}(t, T, x)-b^{*}(t, S, x)\right), b^{*}(t, T, x)\right\rangle \\
& +\int_{\mathbb{R}^{d}}\left(e^{\left\langle b^{*}(t, T, x)-b^{*}(t, S, x), z\right\rangle}-e^{-\left\langle b^{*}(t, S, x), z\right\rangle}+e^{-\left\langle b^{*}(t, T, x), z\right\rangle}-1\right) \nu(d z) \\
& +\int_{\mathcal{I}}\left(e^{c^{*}(t, T, x ; y)-c^{*}(t, S, x ; y)}-e^{-c^{*}(t, S, x ; y)}+e^{-c^{*}(t, T, x ; y)}\right) 1_{\left\{L_{t}+y \leq x\right\}} \nu^{L}(t, d y) .
\end{aligned}
$$

Inserting this in (32) and using the dynamics of $L$ in (31) we obtain the result.

As byproduct of the above proof we obtain the dynamics of the $(S, T, x)$-forward rate.

Corollary 4.3. Assume (A1)-(A4) and (12) holds and $\left(T_{k}, x_{i}\right) \in \mathcal{S}$ with $k<n$. Then, on $\left\{L_{t-\leq x_{i}}\right\}$,

$$
\frac{d F\left(t, T_{k}, T_{k+1}, x_{i}\right)}{F\left(t-, T_{k}, T_{k+1}, x_{i}\right)}=\left(-\lambda\left(t, x_{i}\right)+D\left(t, T_{k}, T_{k+1}, x_{i}\right)\right) d t+d \tilde{\tilde{M}}_{t}
$$

with $D$ from (25) and the local martingale $\tilde{\tilde{M}}$ given in (35).

Proof. Noting that $F\left(t, T_{k}, T_{k+1}, x_{i}\right)=1_{\left\{L_{t} \leq x_{i}\right\}} g\left(t, T_{k}, T_{k+1}, x_{i}\right)$ with $g$ defined in (27), we obtain the dynamics of $F$ via

$$
\begin{aligned}
d F\left(t, T_{k}, x_{i}\right) & =g\left(t-, T_{k}, T_{k+1}, x_{i}\right) d 1_{\left\{L_{t} \leq x_{i}\right\}} \\
& +1_{\left\{L_{t-} \leq x_{i}\right\}} d g\left(t, T_{k}, T_{k+1}, x_{i}\right) \\
& +d\left[1_{\left\{L_{t} \leq x_{i}\right\}}, g\left(t, T_{k}, T_{k+1}, x_{i}\right)\right] .
\end{aligned}
$$


As above we have that

$$
\begin{aligned}
& d\left[1_{\left\{L_{t} \leq x_{i}\right\}}, g\left(t, T_{k}, T_{k+1}, x_{i}\right]\right. \\
& \quad=\int_{\mathcal{I}}\left(1_{\left\{L_{t-}+y \leq x_{i}\right\}}-1_{\left\{L_{t-} \leq x_{i}\right\}}\right) g\left(t-, T_{k}, T_{k+1}, x_{i}\right)\left(e^{C\left(t, T_{k}, T_{k+1} ; y\right)}-1\right) \mu^{L}(d t, d y) .
\end{aligned}
$$

With (30) and (33) we obtain the stated dynamics. Here

$$
\begin{aligned}
d \tilde{\tilde{M}}_{t} & :=d M_{t}^{x}+\int_{\mathbb{R}^{d}}\left(e^{\left\langle b^{*}\left(t, T_{k+1}, x_{i}\right)-b^{*}\left(t, T_{k}, x_{i}\right), z\right\rangle}-1\right)(\mu(d t, d z)-\nu(d z) d t) \\
& +\int_{\mathcal{I}}\left(e^{\left(c^{*}\left(t, T_{k+1}, x_{i} ; y\right)-c^{*}\left(t, T_{k}, x_{i} ; y\right)\right)}-1\right) 1_{\left\{L_{t-}+y \leq x_{i}\right\}}\left(\mu^{L}(d t, d y)-\nu^{L}(d y) d t\right) \\
& +\left\langle b^{*}\left(t, T_{k+1}, x_{i}\right)-b^{*}\left(t, T_{k}, x_{i}\right), d W(t)\right\rangle .
\end{aligned}
$$

\subsection{Forward rate modelling}

In practical applications one needs a simple structure of the $\left(T_{k}, x_{i}\right)$-rates which is analysed in this section. For notational convenience, we consider forward bond prices instead of $\left(T_{k}, x_{i}\right)$-rates themselves. A result for the $\left(T_{k}, x_{i}\right)$-rate can be obtained in a similar way. Recall from (3) that $\lambda(t, x)$ was the intensity that $1_{\left\{L_{t} \leq x\right\}}$ jumps to zero at $t$. Let $\eta(t):=\inf \left\{1 \leq i \leq n: T_{i+1}>t\right\}$. In the following model the forward rate is driven by the Lévy process $Z$ through the functions $\beta_{k}$; the reaction on the loss process $L$ can be adjusted through the functions $\gamma_{k}$. These may depend on the loss occurring at $t, \Delta L_{t}$, and the current loss level $L_{t-}$ itself. We need the following assumption:

(A6) For each $\left(T_{k}, x_{i}\right) \in \mathcal{S}$ the functions $\beta_{k i}: \mathbb{R}^{+} \rightarrow \mathbb{R}^{d}, \gamma_{k i}: \mathbb{R}^{+} \times \mathcal{I} \times[0,1] \rightarrow \mathbb{R}$ are measurable and bounded.

Proposition 4.4. Assume (A1)-(A6) hold. Forward bond prices given on $\left\{L_{t} \leq\right.$ $\left.x_{i}\right\}$ by

$$
\begin{aligned}
& \frac{d F\left(t, T_{k}, T_{k+1}, x_{i}\right)}{F\left(t-, T_{k}, T_{k+1}, x_{i}\right)}=\alpha_{k i}(t) d t+\left\langle\beta_{k i}(t), d W(t)\right\rangle \\
& \quad+\int_{\mathbb{R}^{d}}\left(e^{\left\langle\beta_{k i}(t), z\right\rangle}-1\right) \mu(d t, d z)+\int_{\mathcal{I}}\left(e^{\gamma_{k i}\left(t, L_{t-;} ; y\right)}-1\right) 1_{\left\{L_{t-}+y \leq x_{i}\right\}} \mu^{L}(d t, d y),
\end{aligned}
$$

$\left(T_{k}, x_{i}\right) \in \mathcal{S}, k<n$ and zero on $\left\{L_{t}>x_{i}\right\}$ constitute an arbitrage-free market if

$$
\begin{aligned}
\alpha_{k i}(t) & =-\lambda\left(t, x_{i}\right)+\sum_{j=\eta(t)}^{k}\left\langle\beta_{j i}(t), \Sigma \beta_{k i}(t)\right\rangle \\
& +\int_{\mathbb{R}^{d}}\left(e^{\left\langle\beta_{k i}(t), z\right\rangle}+\left(e^{-\left\langle\beta_{k i}(t), z\right\rangle}-1\right) \prod_{j=\eta(t)}^{k-1} e^{-\left\langle\beta_{j i}(t), z\right\rangle}-1\right) \nu(d z) \\
& +\int_{\mathcal{I}}\left(e^{\gamma_{k i}\left(t, L_{t-;} ; y\right)}+\left(e^{-\gamma_{k i}\left(t, L_{t-} ; y\right)}-1\right) \prod_{j=\eta(t)}^{k-1} e^{-\gamma_{j i}\left(t, L_{t-} ; y\right)}\right) 1_{\left\{L_{t}+y \leq x_{i}\right\}} \nu^{L}(t, d y)
\end{aligned}
$$

for all $\left(T_{k}, x_{i}\right),\left(T_{k+1}, x_{i}\right) \in \mathcal{S}$. 
In this way the $(T, x)$-forward bond price explicitly relates to $\lambda(t, x)$ and $\nu^{L}$. With this result at hand pricing of typical derivatives on the forward rate can be done in a standard way. The input parameters for the modeler are $\beta_{k i}, \gamma_{k i}$ as well as $\Sigma, \nu$ and $\nu^{L}$ while the $\alpha_{k i}$ are determined as above to ensure that the model is free of arbitrage.

Proof. The idea is to identify $b$ (see (6)) from the dynamics of $F$ and then show that the drift condition (12) holds with the chosen $\alpha$ : a comparison of (36) with Corollary 4.3 and (35) yields that

$$
\beta_{k i}(t)=\int_{T_{k}}^{T_{k+1}} b\left(t, u, x_{i}\right) d u \quad \text { and } \quad \gamma_{k i}\left(t, L_{t-} ; y\right)=\int_{T_{k}}^{T_{k+1}} c\left(t, u, x_{i} ; y\right) d u .
$$

The drift in (34) yields that

$$
\begin{aligned}
\alpha_{k i}(t) & =-\lambda\left(t, x_{i}\right)+\left\langle\Sigma \beta_{k i}(t), b^{*}\left(s, T_{k+1}, x_{i}\right)\right\rangle \\
& +\int_{\mathbb{R}^{d}}\left(e^{\left\langle\beta_{k i}(t), z\right\rangle}+e^{-\left\langle b^{*}\left(t, T_{k}, x_{i}\right), z\right\rangle}\left(e^{-\left\langle\beta_{k i}(t), z\right\rangle}-1\right)-1\right) \nu(d z) \\
& +\int_{\mathcal{I}}\left(e^{\gamma_{k i}\left(y, L_{t-}\right)}+e^{-c^{*}\left(t, T_{k}, x_{i} ; y\right)}\left(e^{-\gamma_{k i}\left(y, L_{t-}\right)}-1\right)\right) 1_{\left\{L_{t}+y \leq x_{i}\right\}} \nu^{L}(d y) .
\end{aligned}
$$

We have that $\beta_{k i}(t)=b^{*}\left(t, T_{k+1}, x_{i}\right)-b^{*}\left(t, T_{k}, x_{i}\right)$ and we use the freedom to specify $b\left(t, T, x_{i}\right) \equiv 0$ whenever $t>T$. This in turn gives that for $T_{j}<t<T_{j+1}$

$$
\beta_{j i}(t)=\int_{T_{j}}^{T_{j+1}} b\left(t, u, x_{i}\right) d u=\int_{t}^{T_{j+1}} b\left(t, u, x_{i}\right) d u=b^{*}\left(t, T_{j+1}, x_{i}\right)
$$

such that

$$
b^{*}\left(t, T_{k+1}, x_{i}\right)=\sum_{j=\eta(t)}^{k} \beta_{j i}(t)
$$

Analogously,

$$
c^{*}\left(t, T_{k+1}, x_{i} ; y\right)=\sum_{j=\eta(t)}^{k} \gamma_{j i}\left(t, L_{t-} ; y\right) .
$$

Inserting this in (37) gives the claim.

\section{$4.3\left(T_{k}, x_{i}\right)$-rate modelling}

In this section we study the case where instead of the forward price process the $\left(T_{k}, x_{i}\right)$-rate has a simple structure. As in the previous setting we consider the lognormal case including jumps. For simplicity we assume $\nu=0$, i.e. the dynamics is only driven by a Brownian motion and $L$. In turn we will obtain the result from Brace, Gatarek, and Musiela (1997) as a special case. Finally, we also give the results for market models in a single-name credit risky case.

(A7) Assume $\nu(d x)=0$ and that for each $\left(T_{k}, x_{i}\right) \in \mathcal{S}$ the functions $\beta_{k i}: \mathbb{R}^{+} \rightarrow \mathbb{R}^{d}$, $\gamma_{k i}: \mathbb{R}^{+} \times \mathbb{R}^{+} \rightarrow \mathbb{R}$ are measurable and bounded. 
Proposition 4.5. Assume (A1)-(A4) and (A7) hold. $\left(T_{k}, x_{i}\right)$-rates given by

$$
\begin{aligned}
d L\left(t, T_{k}, x_{i}\right) & =L\left(t-, T_{k}, x_{i}\right)\left(\alpha_{k i}(t) d t+\left\langle\beta_{k i}, d W(t)\right\rangle\right) \\
& +\frac{1+\delta_{k} L\left(t-, T_{k}, x_{i}\right)}{\delta_{k}} \int_{\mathcal{I}}\left(e^{\gamma_{k i}\left(y, L_{t-}\right)}-1\right) 1_{\left\{L_{t-}+y \leq x_{i}\right\}} \mu^{L}(d s, d y)
\end{aligned}
$$

for all $\left(T_{k}, x_{i}\right) \in \mathcal{S}$ and zero otherwise constitute an arbitrage-free market if

$$
\begin{aligned}
\alpha_{k i}(t)= & -\lambda\left(t, x_{i}\right)+\sum_{j=\eta(t)}^{k} \frac{1+\delta_{j} L\left(t-, T_{j}, x_{i}\right)}{\delta_{j} L\left(t-, T_{j}, x_{i}\right)}\left\langle\beta_{j i}(t), \Sigma \beta_{k i}(t)\right\rangle \\
+ & \frac{1+\delta_{k} L\left(t-, T_{k}, x_{i}\right)}{\delta_{k} L\left(t-, T_{k}, x_{i}\right)} \\
& \cdot \int_{\mathcal{I}}\left(e^{\gamma_{k i}(t ; y)}+\left(e^{-\gamma_{k i}\left(t, L_{t-} ; y\right)}-1\right) \prod_{j=\eta(t)}^{k-1} e^{-\gamma_{j i}\left(t, L_{t-;}\right)}\right) 1_{\left\{L_{t}+y \leq x_{i}\right\}} \nu^{L}(d y)
\end{aligned}
$$

for all $\left(T_{k}, x_{i}\right) \in \mathcal{S}$.

With this result at hand pricing of typical derivatives on the $\left(T_{k}, x_{i}\right)$-rates can be done in a standard way, see for example Section 6 in Brace, Gatarek, and Musiela (1997). As previously, the input parameters for the modeler are $\lambda, \beta_{k i}$ and $\gamma_{k i}$ while the $\alpha_{k i}$ are determined as above to ensure that the model is free of arbitrage.

Proof. We proceed similar to the proof of Proposition 4.4. A comparison of (40) with (26) yields that

$$
\beta_{k i}(t)=\frac{1+\delta_{k} L\left(t-, T_{k}, x_{i}\right)}{\delta_{k} L\left(t-, T_{k}, x_{i}\right)}\left(b^{*}\left(t, T_{k+1}, x_{i}\right)-b^{*}\left(t, T_{k}, x_{i}\right)\right)
$$

such that

$$
\begin{aligned}
b^{*}\left(t, T_{k+1}, x_{i}\right) & =b^{*}\left(t, T_{k}, x_{i}\right)+\beta_{k i}(t) \frac{\delta_{k} L\left(t-, T_{k}, x_{i}\right)}{1+\delta_{k} L\left(t-, T_{k}, x_{i}\right)} \\
& =\sum_{j=\eta(t)}^{k} \beta_{j i}(t) \frac{\delta_{j} L\left(t-, T_{j}, x_{i}\right)}{1+\delta_{j} L\left(t-, T_{j}, x_{i}\right)}
\end{aligned}
$$

compare (38). Furthermore, we have that $\gamma_{k i}\left(y, L_{t-}\right)=C\left(t, T_{k}, T_{k+1} x_{i} ; y\right)$ and hence (39). From the drift term in (24) we obtain that

$$
\alpha_{k i}(t)=-\lambda\left(t, x_{i}\right)+\frac{1+\delta_{k} L\left(t-, T_{k}, x_{i}\right)}{\delta_{k} L\left(t-, T_{k}, x_{i}\right)} D\left(t, T_{k}, T_{k+1}, x_{i}\right) .
$$

With (42) and (39)

$$
\begin{aligned}
& D\left(t, T_{k}, T_{k+1}, x_{i}\right)=\frac{\delta_{k} L\left(t-, T_{k}, x_{i}\right)}{1+\delta_{k} L\left(t-, T_{k}, x_{i}\right)}\left\langle\Sigma \beta_{k i}(t), b^{*}\left(t, T_{k+1}, x_{i}\right)\right\rangle \\
& \quad+\int_{\mathcal{I}}\left(e^{\gamma_{k i}\left(t, L_{t-} ; y\right)}+\left(e^{-\gamma_{k i}\left(t, L_{t-} ; y\right)}-1\right) \prod_{j=\eta(t)}^{k-1} e^{-\gamma_{j i}\left(t, L_{t-} ; y\right)}\right) 1_{\left\{L_{t}+y \leq x_{i}\right\}} \nu^{L}(d y)
\end{aligned}
$$

and we conclude as in Proposition 4.4 . 
Remark 4.6. With $x_{m}=1$ and $c(\cdot, T, 1 ; y)=1$ (from Assumption (A2)) gives the risk-free Libor market model as a special case and (41) equals Equation (2.6) in Brace, Gatarek, and Musiela (1997). Also a doubly stochastic model for singlename credit risk as in Huehne (2007) is a special case: consider the doubly stochastic random time $\tau$ which has an intensity $\left(\lambda_{t}\right)_{t \geq 0}$. Choose $L_{t}:=\frac{1}{2} 1_{\{\tau \leq t\}}$. Then, with $x_{1}=\frac{1}{2}$, one has that the rates of defaultable bond satisfying, on $\{\tau>t\}$,

$$
\frac{d \bar{L}\left(t, T_{k}\right)}{\bar{L}\left(t-, T_{k}\right)}=\bar{\alpha}_{k}(t) d t+\left\langle\bar{\beta}_{k}(t), d W(t)\right\rangle
$$

and zero otherwise constitute an arbitrage-free market if

$$
\bar{\alpha}_{k}(t)=-\lambda(t)+\left\langle\bar{\beta}_{k}(t), \sum_{j=\eta(t)}^{k} \frac{\delta_{j} \bar{L}\left(t-, T_{j}\right)}{1+\delta_{j} \bar{L}\left(t-, T_{j}\right)} \bar{\beta}_{j}(t)\right\rangle .
$$

\section{References}

Björk, T., G. B. Di Masi, Y. Kabanov, and W. J. Runggaldier (1997). Towards a general theory of bond markets. Finance and Stochastics, 141-174.

Brace, A., D. Gatarek, and M. Musiela (1997). The market model of interest rate dynamics. Mathematical Finance 7, 127-155.

Eberlein, E., Z. Grbac, and T. Schmidt (2010). Market models driven by Lévy processes. Working paper.

Eberlein, E., W. Kluge, and P. J. Schönbucher (2006). The Lévy Libor model with default risk. Journal of Credit Risk 2(2), 3-42.

Eberlein, E. and F. Özkan (2005). The Lévy LIBOR model. Finance and Stochastics 9, $327-348$.

Filipović, D. (2001). Consistency Problems for Heath-Jarrow-Morton Interest Rate Models, Volume 1760 of Lecture Notes in Mathematics. Springer Verlag. Berlin Heidelberg New York.

Filipović, D. (2009). Term Structure Models: A Graduate Course. Springer Verlag. Berlin Heidelberg New York.

Filipović, D., L. Overbeck, and T. Schmidt (2010). Dynamic CDO term structure modelling. Mathematical Finance, Forthcoming.

Heath, D., R. A. Jarrow, and A. J. Morton (1992). Bond pricing and the term structure of interest rates. Econometrica 60, 77-105.

Huehne, F. (2007). Defaultable Lévy Libor rates and credit derivatives. International Journal of Theoretical and Applied Finance 10, $407-435$.

Jakubowski, J. and J. Zabczyk (2007). Exponential moments for HJM models with jumps. Finance Stoch. 11(3), 429-445.

Musiela, M. and M. Rutkowski (1997). Martingale Methods in Financial Modelling. Springer, Berlin.

Özkan, F. and T. Schmidt (2005). Credit risk with infinite dimensional Lévy processes. Statistics and Decisions 23, 281 - 299.

Schmidt, T. (2006). An infinite factor model for credit risk. International Journal of Theoretical and Applied Finance 9, 43-68.

Schmidt, T. and S. Tappe (2010). General cdo term structure modelling: existence and monotonicity. Working paper. 